\title{
cRGD-functionalized, DOX-conjugated, and ${ }^{64} \mathrm{Cu}$-labeled superparamagnetic iron oxide nanoparticles for targeted anticancer drug delivery and PET/MR imaging
}

\author{
Xiaoqiang Yang ${ }^{a}$, Hao Hong ${ }^{b}$, Jamison J. Grailer ${ }^{c}$, lan J. Rowland ${ }^{d}$, Alireza Javadie, Samuel \\ A. Hurley ${ }^{d}$, Yuling Xiao ${ }^{e}$, Yunan Yang ${ }^{b}$, Yin Zhang ${ }^{b}$, Robert J. Nickles ${ }^{b}$, Weibo Cai ${ }^{b,}{ }^{,}$, \\ Douglas A. Steeber ${ }^{\mathrm{C}}$, and Shaoqin Gonge, \\ aDepartment of Mechanical Engineering, University of Wisconsin-Milwaukee, Milwaukee, WI \\ 53211. USA \\ 'Department of Biological Science, University of Wisconsin-Milwaukee, Milwaukee, WI 53211. \\ USA \\ bDepartments of Radiology and Medical Physics, University of Wisconsin - Madison, Madison, \\ WI, USA \\ dDepartment of Medical Physics, University of Wisconsin-Madison, Madison, WI 53706, USA \\ eDepartment of Biomedical Engineering and Wisconsin Institutes for Discovery, University of \\ Wisconsin-Madison, Madison, WI 53706, USA
}

\begin{abstract}
Multifunctional and water-soluble superparamagnetic iron oxide (SPIO) nanocarriers were developed for targeted drug delivery and positron emission tomography/magnetic resonance imaging (PET/MRI) dual-modality imaging of tumors with integrin $\alpha_{\mathrm{v}} \beta_{3}$ expression. An anticancer drug was conjugated onto the PEGylated SPIO nanocarriers via pH-sensitive bonds. Tumor targeting ligands, cyclo(Arg-Gly-Asp-D-Phe-Cys) (c(RGDfC)) peptides, and PET ${ }^{64} \mathrm{Cu}$ chelators, macrocyclic 1,4,7-triazacyclononane- $\mathrm{N}, \mathrm{N}^{\prime}, \mathrm{N}^{\prime \prime}$-triacetic acid (NOTA), were conjugated onto the distal ends of the PEG arms. The effectiveness of the SPIO nanocarriers as an MRI contrast agent was evaluated via an in vitro $r_{2}$ MRI relaxivity measurement. $c R G D$-conjugated SPIO nanocarriers exhibited a higher level of cellular uptake than cRGD-free ones in vitro. Moreover, cRGD-conjugated SPIO nanocarriers showed a much higher level of tumor accumulation than cRGD-free ones according to noninvasive and quantitative PET imaging, and ex vivo biodistribution studies. Thus, these SPIO nanocarriers demonstrated promising properties for combined targeted anticancer drug delivery and PET/MRI dual-modality imaging of tumors. Keywords: superparamagnetic iron oxide; drug delivery; Positron Emission Tomography (PET); Magnetic Resonance Imaging (MRI); nanomedicine
\end{abstract}

\section{Introduction}

Chemotherapy is frequently used to treat cancer patients. However, systemically administered chemotherapeutic agents exhibit poor specificity in reaching tumor tissues and thus can cause serious adverse effects. Many of the pharmacological properties of conventional ("free") drugs can be improved through the use of drug nanocarriers, including 
poor solubility in aqueous media, poor biodistribution, unfavorable pharmacokinetics, and lack of selectivity for target tissues [1].

In recent years, various types of drug/agent nanocarriers have been investigated, among which a small number of such systems are already commercialized or are in clinical studies [2]. Desirable functionalities for next-generation, multifunctional drug/agent nanocarriers include: (1) excellent in vivo stability before reaching the target sites; (2) high drug loading content; (3) relatively long circulation time in the bloodstream; (4) ability to accumulate specifically in the target sites; (5) ability to influence the intracellular drug/agent uptake behavior; (6) responsiveness to local stimuli such as changes in $\mathrm{pH}$ and/or temperature to provide controlled release; (7) ability to observe its accumulation inside the target sites in real-time; and (8) ability to monitor disease progression. Although a number of drug/agent nanocarriers reported in the literature have demonstrated a variety of useful properties, multifunctional nanocarriers simultaneously exhibiting these eight functionalities are extremely rare [3-7]. Multifunctional nanoparticles (NPs) that incorporate therapeutic agents, molecular targeting, and diagnostic imaging capabilities and exhibit desirable characteristics for in vivo applications can greatly enhance the efficacy of cancer therapy and accuracy of diagnosis/prognosis which can pave the road for personalized medicine [8$11]$.

Although many molecular imaging modalities are available in the clinic today, there is not a single modality that is perfect and sufficient to obtain all necessary information for a particular question. For instance, positron emission tomography (PET) has excellent sensitivity but with relatively poor spatial resolution. Over the last decade, positron emission tomography/computer tomography (PET/CT) dual-modality imaging has become a gold standard in clinical oncology because it can provide both molecular (PET) and anatomical (CT) information [12-14]. However, accurate localization of PET tracer uptake, even with $\mathrm{PET} / \mathrm{CT}$, can be very difficult in some cases due to the absence of identifiable anatomical structure (e.g., in the abdomen). Moreover, CT imaging may expose patients to a substantial amount of ionizing radiation. Magnetic resonance imaging (MRI), on the other hand, does not require ionizing radiation and can provide excellent spatial resolution and soft tissue contrast which is superior to CT $[15,16]$. Thus, PET/MRI has the potential to become the imaging modality of choice for various clinical applications such as neurological studies, certain types of cancer, and the emerging field of stem cell therapy $[17,18]$.

The wide-spread use of PET/MRI scanners will greatly benefit from the use of agents that incorporate both PET isotopes and MRI contrast agents [19]. Multimodality imaging using a small molecule-based probe is very challenging due to the limited number of attachment points and the potential steric interference with receptor binding. On the other hand, nanoparticles (NPs) have large surface areas where multiple functional moieties can be incorporated for multimodality molecular imaging [20,21]. For instance, MRI using superparamagnetic iron oxide (SPIO) NPs as contrast agents has been applied to detect apoptosis, angiogenesis, and tissue infiltration during the development of cancer, among others [22].

In this paper, we report a multifunctional and water-soluble SPIO NP-based nanomedicine that can potentially exhibit all eight functionalities as described earlier. The therapeutic drug, doxorubicin (DOX), was conjugated onto the SPIO nanocarriers using a pH-sensitive hydrazone bond to achieve $\mathrm{pH}$-responsive drug release. Integrin $\alpha_{\mathrm{v}} \beta_{3}$, a cell-adhesion molecule that is expressed on both proliferating tumor vasculature and certain tumors cells but not on normal vasculature/cells [23,24] was chosen as the molecular target in this study. Cyclic arginine-glycine-aspartic acid (cRGD) peptide, a potent integrin $\alpha_{v} \beta_{3}$ antagonist, was conjugated onto the SPIO nanocarriers to enhance their tumor targeting ability. Aside from 
its ability to deliver and release the anticancer drug more specifically at the tumor vasculature and cells, these multifunctional SPIO nanocarriers can also carry PET isotopes (e.g., ${ }^{64} \mathrm{Cu}, \mathrm{t}_{1 / 2}=12.7 \mathrm{~h}$ ) via a macrocyclic chelating agent (i.e., 1,4,7-triazacyclononane-N, $\mathrm{N}^{\prime}, \mathrm{N}^{\prime \prime}$-triacetic acid [NOTA]) conjugated onto the SPIO nanocarriers, thereby making it possible to use PET to non-invasively and quantitatively monitor the biodistribution, pharmacokinetics, and tumor targeting efficacy of the drug nanocarriers. In addition, the SPIO core can significantly enhance the sensitivity of MRI detection of the drug nanocarriers at the tumor sites thereby making it possible to accurately monitor the anticancer effects of the nanocarriers in vivo. The use of such multifunctional SPIO nanocarriers in clinics in the future would allow physicians to predict the therapeutic effects (e.g., based on the amount of drug accumulation in the tumor site) and monitor the cancer progression in individual patients thereby paving the road for personalized medicine.

\section{Materials and methods}

\subsection{Materials}

The heterobifunctional poly(ethylene glycol) (PEG) derivatives, $\mathrm{R}(\mathrm{R}=$ maleimide or methoxy)-PEG 114 -NHS ( $N$-hydroxysuccinimide) (Mw:5000) were purchased from JenKem Technology, USA. The anticancer drug, doxorubicin $\mathrm{HCl}$, was purchased from Tecoland Corporation, USA. Mono-ethyl malonate (MEM), anhydrous hydrazine, benzyl ether (99 $\%$ ), oleylamine (>70 \%), 1-ethyl-3-(3-dimethylaminopropyl) carbodiimide (EDC) and iron(III) acetylacetonate were purchased from Sigma-Aldrich, USA. Cyclo (Arg-Gly-AspD-Phe-Cys) (c(RGDfC)) peptide was purchased from Creative Peptides in USA. P-SCN-BnNOTA was purchased from Macrocyclics, Inc. (Dallas, TX). All other chemicals used were of analytical reagent grade. Phosphate and acetate buffered solutions were prepared in our laboratory. DMEM was purchased from Gibco BRL, USA. U87MG human glioblastoma cells were purchased from ATCC and were cultured in DMEM supplemented with $10 \%$ fetal calf serum.

\subsection{Synthesis of $10 \mathrm{~nm}$ hydrophobic SPIO NPs}

The SPIO NPs were synthesized following a previously reported method [25]. Briefly, 2 mmol of $\mathrm{Fe}(\mathrm{acac})_{3}$ was dissolved in a mixture of $10 \mathrm{~mL}$ benzyl ether and $10 \mathrm{~mL}$ oleylamine. The solution was dehydrated at $110{ }^{\circ} \mathrm{C}$ for $1 \mathrm{~h}$, and was quickly heated to $300{ }^{\circ} \mathrm{C}$ and kept at this temperature for $2 \mathrm{~h}$. After cooling, $50 \mathrm{~mL}$ of ethanol was added into the solution. The precipitation was collected by centrifuge at $6000 \mathrm{rpm}$ and was washed in ethanol three times. Finally, the product was redispersed in hexane.

\subsection{Synthesis of nitrodopamine (NDA)}

Nitrodopamine was synthesized according to a previously published procedure [26,27]. Briefly, dopamine hydrochloride $(1.90 \mathrm{~g}, 10 \mathrm{mmol})$ and sodium nitrite $(1.52 \mathrm{~g}, 22 \mathrm{mmol})$ were dissolved in water $(25 \mathrm{~mL})$ and cooled down to $0{ }^{\circ} \mathrm{C}$. Sulfuric acid $(17.4 \mathrm{mmol}$ in 10 $\mathrm{mL}$ water) was added slowly to the mixture and a yellow precipitate was formed. After stirring at room temperature overnight, the precipitate was filtered and recrystallized from water. The product was dried under high vacuum to give nitrodopamine.

\subsection{Synthesis of NDA anchored hydrophobic SPIO NPs (SPIO-NDA)}

The NDA anchored SPIO NPs were synthesized using the method described by Xie et al. with slight modification [25]. Briefly, in $3 \mathrm{~mL} \mathrm{CHCl}_{3}$ solution containing $43 \mathrm{mg}$ of $10 \mathrm{~nm}$ hydrophobic $\mathrm{Fe}_{3} \mathrm{O}_{4} \mathrm{NPs}, 38 \mathrm{mg}$ of NDA dissolved in $2 \mathrm{~mL}$ DMSO was added into the above solution dropwise over 5 minutes with stirring at room temperature. After an overnight reaction, the precipitate was separated using a magnet and washed with DMSO 
twice followed by centrifuge. Finally, the SPIO-NDA NPs were redispersed into DI water and purified by dialyzing against DI water.

\subsection{Determination of the number of NDA on the surface of each SPIO NPs}

We measured the weight of NPs that were transferred into the aqueous phase and the amounts of NDA that were taken up by the magnetic NPs. Using the size composition of the NPs, we estimated that the number of NDA on the surface of each nanoparticle was 1820.

\subsection{Synthesis of $\mathrm{N}$-hydroxysucinimide ester of mono-ethyl malonate (MEM-NHS)}

$1.34 \mathrm{mg}$ of EDC $(0.007 \mathrm{mmol})$ and $0.8 \mathrm{mg}$ of NHS $(0.007 \mathrm{mmol})$ were added to $1 \mathrm{~mL}$ DI water containing $1.85 \mathrm{mg}$ of MEM $(0.014 \mathrm{mmol})$. The reaction was allowed to stay at room temperature for $5 \mathrm{~h}$ under stirring to obtain MEM-NHS.

\subsection{Conjugation of the $\mathrm{N}$-hydroxysucinimide ester of methoxy poly(ethylene glycol) (MPEG-NHS) (Mw: 5,000), NHS ester of maleimide poly(ethylene glycol) (Mal-PEG-NHS) (Mw:5000) and NHS ester of mono-ethyl malonate (MEM-NHS) onto the surface of SPIO NPs}

Since the number of NDA anchored onto each SPIO NP was estimated to be 1820, the molar ratio for MEM-NHS:MEPG-NHS:Mal-PEG-NHS was set at 910:364:546 initially. The 910 MEM molecules allow up to 910 DOX molecules to be conjugated onto each SPIO NP via pH-sensitive hydrazone bonds. The 364 MPEG arms per SPIO NP were used to adjust its solubility/stability in physiological conditions and help to control the molar ratio of cRGD and NOTA. The 546 Mal-PEG arms were used to conjugate the cRGD tumor-targeting ligands and the isotope ${ }^{64} \mathrm{Cu}$ chelators (i.e., 1,4,7-triazacyclononane-1,4,7-triacetic acid (NOTA)). The molar ratio for cRGD and NOTA was set at $40 \%$ and $20 \%$ of the total number of PEG arms, respectively, leading to 364 arms for cRGD conjugation and 182 arms for NOTA conjugation. Briefly, $3.46 \mathrm{mg}$ MEM-NHS (0.014 mmol), $30 \mathrm{mg}$ MPEG-NHS $(0.006 \mathrm{mmol})$ and $40 \mathrm{mg}$ Mal-PEG-NHS $(0.008 \mathrm{mmol})$ were added to $10 \mathrm{~mL}$ DI water containing $30 \mathrm{mg}$ SPIO-NDA NPs under a $\mathrm{N}_{2}$ atmosphere. The mixture was allowed to react at room temperature for $5 \mathrm{~h}$ after $30 \mathrm{~min}$ of $\mathrm{N}_{2}$ bubbling. After the reaction was complete, the solution was purified by dialysis against DI water for 2 days. The solution was then filtered through a $0.22 \mu \mathrm{m}$ membrane followed by freeze-drying.

\subsection{Conjugation of the DOX molecules onto the SPIO NPs}

The DOX molecules were conjugated onto the SPIO NPs via a two-step reaction procedure. Briefly, in the first step, the methoxy group of MEM was substituted with hydrazide by an ester-amide exchange aminolysis reaction in anhydrous DMSO at $40{ }^{\circ} \mathrm{C}$ for $24 \mathrm{~h}$. The resulting hydrazine-modified SPIO NPs were purified by extensive dialysis against $0.25 \%$ ammonia solution for $48 \mathrm{~h}$ followed by freeze-drying. In the second step, DOX was conjugated to the hydrazide group of the SPIO NP through an acid-sensitive hydrazone linkage. The reaction was conducted in DMSO solution at room temperature for $24 \mathrm{~h}$ with an excess amount of DOX. The unbound DOX was removed first by dialysis against DI water and then purified over a Sephadex LH-20 gel column.

\section{9. $\mathrm{CRGD}$ and NOTA conjugation onto the NPs}

The cRGD and NOTA conjugation onto the NPs was achieved by a reaction between the maleimide group of the PEG conjugated on the surface of SPIO NPs and the thiol group of the cRGD and thiol-functionalized NOTA (NOTA-SH). The NOTA-SH was prepared by a reaction between the NCS group of p-SCN-Bn-NOTA and the amino group of 2aminoethanethiol hydrocholoride in the presence of triethanolamine. The reaction was allowed to stay in DI water at room temperature for $3 \mathrm{~h}$ under a $\mathrm{N}_{2}$ atmosphere. Thereafter, a 
predetermined amount of cRGD and NOTA-SH water solution were added into the water solution containing the PEGylated DOX-conjugated SPIO NPs. The molar ratio for cRGD:NOTA was set at 2:1, and the total molar value was equal to that of Mal-PEG. This reaction was carried out in water solution at room temperature for $5 \mathrm{~h}$ under a $\mathrm{N}_{2}$ atmosphere. After the reaction was complete, the solution was purified by dialysis against DI water for 2 days.

\subsection{Determine the content of DOX and Fe in the SPIO nanocarriers}

The DOX-loading content (DLC), defined as the weight percentage of DOX in the SPIO nanocarriers, was quantified by UV-Vis analysis using a Varian Cary model 100 Bio UVVis spectrophotometer. First, DOX was released from the SPIO nanocarriers in a $0.1 \mathrm{~N} \mathrm{HCl}$ solution to cleave the hydrazone bonds. The absorbance of DOX at $485 \mathrm{~nm}$ was measured to determine the drug content in the solution using a previously established calibration curve. The DLC measurements were performed in triplicate for each of the samples. The weight percentage of iron in the SPIO nanocarriers was determined using an atomic absorption spectrophotometer (AAS). Briefly, the freeze-dried samples were weighed and then added to a $1 \mathrm{M} \mathrm{HCl}$ solution to allow the complete dissolution of the SPIO NPs. Iron concentration was determined at the specific Fe absorption wavelength $(248.3 \mathrm{~nm})$ based on a previously established calibration curve.

\subsection{Characterization}

The size and size distribution of the SPIO nanocarriers were determined by dynamic light scattering (DLS) (Malvern Zetasizer Nano Instrument, USA) with an angle detection of $90^{\circ}$. Samples for transmission electron microscopy (TEM, Hitachi H-600) analysis were prepared by drying a dispersion of the SPIO nanocarriers on a copper grid coated with amorphous carbon.

\subsection{Evaluation of $\mathrm{pH}$-controlled drug release}

The $\mathrm{pH}$-responsive DOX release behavior of the SPIO nanocarriers was studied using an UV-spectrophotometer. Briefly, $50 \mathrm{mg}$ of freeze-dried cRGD/DOX-conjugated SPIO nanocarriers were dispersed in $5 \mathrm{~mL}$ of medium (phosphate buffer (10 mM, pH 7.4) or acetate buffer $(10 \mathrm{mM}, \mathrm{pH} 5.3))$ and were then placed in a dialysis bag with a molecular weight cut-off of $2 \mathrm{kDa}$. Subsequently, the dialysis bag was immersed in $100 \mathrm{~mL}$ of the same medium and kept in a horizontal laboratory shaker maintaining a constant temperature and stirring. At selected time intervals, the buffered solution $(5 \mathrm{~mL})$ outside the dialysis bag was removed for UV-Vis analysis and replaced by an equal volume of fresh-buffered solution. The amount of released DOX was analyzed with the UV spectrophotometer at 485 $\mathrm{nm}$. Each sample was measured three times.

\subsection{Cellular uptake study}

The cellular uptake behavior and the intracellular distribution of the nanocarriers were analyzed using both flow cytometry and confocal laser scanning microscopy (CLSM). For flow cytometry, U87MG cells $\left(1 \times 10^{6}\right)$ were seeded in 6-well culture plates and cultured in DMEM overnight. The cells were then treated with free DOX, and cRGD-conjugated and cRGD-free SPIO nanocarriers for 15 and $120 \mathrm{~min}$ (DOX concentration $20 \mu \mathrm{g} / \mathrm{mL}$ ), respectively. Thereafter, the cells were lifted using Cellstripper ${ }^{\mathrm{TM}}$ (Media Tech, Inc.) and washed. DOX uptake was analyzed using a FACSCalibur flow cytometer and CellQuest Pro software (BD Bioscience). A minimum of $2 \times 10^{4}$ cells were analyzed from each sample with the DOX fluorescence intensity shown on a four-decade log scale. For CLSM studies, U87MG cells $\left(1 \times 10^{6}\right)$ were seeded onto $22 \mathrm{~mm}$ round glass coverslips placed in 6-well plates and cultured overnight. Cells were treated with free DOX, and cRGD-conjugated and 
cRGD-free SPIO nanocarriers for $120 \mathrm{~min}$ (DOX concentration: $20 \mu \mathrm{g} / \mathrm{mL}$ ). Following incubation, the cells were washed and fixed with $1.5 \%$ formaldehyde. Coverslips were placed onto glass microscope slides mounted in Prolong Gold ${ }^{\circledR}$ mounting medium (Invitrogen) and DOX uptake was analyzed using a Leica TCS SP2 Confocal System installed on an upright compound microscope (Leica, Wetzler, Germany). Digital monochromatic images were acquired using Leica Confocal Software (Version 2.61).

\subsection{Cytotoxicity evaluation}

The cytotoxicity of the SPIO nanocarriers against U87MG cells was studied using the MTT assay. First, U87MG cells $\left(1 \times 10^{4}\right)$ were seeded in 96-well plates and incubated for $24 \mathrm{~h}$ in DMEM. The media was replaced with fresh media containing either free DOX, or cRGDconjugated or cRGD-free SPIO nanocarriers at different DOX concentrations $(10,20$, and $40 \mu \mathrm{g} / \mathrm{mL}$ ) or control media and incubated for $48 \mathrm{~h}$. Thereafter, the wells were washed three times with warm phosphate buffer solution and incubated again for another $4 \mathrm{~h}$ with DMEM containing $250 \mu \mathrm{g} / \mathrm{mL}$ of MTT. After discarding the culture medium, $200 \mu \mathrm{l}$ of DMSO was added to dissolve the precipitates and the resulting solution was measured for absorbance at $570 \mathrm{~nm}$ with a reference wavelength of $690 \mathrm{~nm}$ using a microtiter plate reader (Molecular Devices). Significant differences in cytotoxicity between treatment groups were determined using a two-tailed Student's $t$-test with $\mathrm{p}<0.05$ being considered significant.

\subsection{Relaxivity measurement}

All samples were positioned in a Varian (Palo Alto, California, USA) $7.2 \mathrm{~cm}$ inner diameter quadrature coil and relaxation data were acquired at $4.7 \mathrm{~T}$ using a Varian Inova imaging and spectroscopy system. A single slice, multi-echo spin echo sequence was used to measure $\mathrm{T}_{2}$ relaxation times $(\mathrm{TR}=8000 \mathrm{~ms}, \mathrm{TE}=5.75-20.75 \mathrm{~ms}$ ( 16 echoes, $1 \mathrm{~s}$ increments), $\mathrm{SL}=2 \mathrm{~mm}$, $\mathrm{FOV}=60 \times 60 \mathrm{~mm}, \mathrm{MA}=128 \times 128)$. Relaxation times were obtained by fitting the multi-echo data to a monoexponential decay curve using linearized least-squares optimization. Relaxivity values were calculated via linear least-squares fitting of 1/relaxation time $\left(\mathrm{s}^{-1}\right)$ versus the iron concentration $(\mathrm{mM} \mathrm{Fe})$.

\subsection{Animal model}

All animal studies were conducted under a protocol approved by the University of Wisconsin Institutional Animal Care and Use Committee. U87MG cells were used for tumor inoculation when they reached $\sim 80 \%$ confluence. Four- to five-week-old female athymic nude mice were purchased from Harlan (Indianapolis, IN) and tumors were established by subcutaneously injecting $5 \times 10^{6}$ cells, suspended in $100 \mu \mathrm{L}$ of $1: 1$ mixture of medium and matrigel (BD Biosciences, Franklin lakes, NJ), into the front flank of mice [28]. The tumor sizes were monitored every other day and the animals were subjected to in vivo experiments when the diameter of the tumors reached 6 8mm (typically 4 weeks after inoculation).

\subsection{7. ${ }^{64} \mathrm{Cu}$-labeling of SPIO nanocarriers}

${ }^{64} \mathrm{Cu}$-labeling was performed similar to previously published procedures [29]. In brief, ${ }^{64} \mathrm{CuCl}_{2}$ (74 MBq) was diluted in $300 \mu \mathrm{L}$ of $0.1 \mathrm{M}$ sodium acetate buffer ( $\mathrm{pH} 6.5$ ) and added to the cRGD-conjugated or cRGD-free SPIO nanocarriers solution containing approximately $6 \mathrm{ng}$ of NOTA. The reaction mixture was incubated for $40 \mathrm{~min}$ at $40^{\circ} \mathrm{C}$ with constant shaking. The ${ }^{64} \mathrm{Cu}$-labeled cRGD-conjugated or cRGD-free SPIO nanocarriers were purified using PD-10 columns with phosphate-buffered saline (PBS) as the mobile phase before animal studies. 


\subsection{MicroPET imaging}

PET scans were performed using a microPET/microCT Inveon rodent model scanner (Siemens Medical Solutions USA, Inc.). U87MG tumor-bearing mice were injected with 5$10 \mathrm{MBq}$ of ${ }^{64} \mathrm{Cu}$-labeled cRGD-free or cRGD-conjugated SPIO nanocarriers via tail vein and 3-15 min static PET scans were performed at various time points p.i. The images were reconstructed using a Bayesian maximum a posteriori (MAP) algorithm, with no attenuation or scatter correction. For each microPET scan, three-dimensional (3D) ROIs were drawn over the tumor and major organs by using the vendor software (Inveon Research Workshop) on decay-corrected whole-body coronal images. Assuming a tissue density of $1 \mathrm{~g} / \mathrm{mL}$, the ROIs were converted to $\mathrm{MBq} / \mathrm{g}$ using a conversion factor (pre-determined using a $50 \mathrm{~mL}$ centrifuge tube filled with ${ }^{64} \mathrm{CuCl}_{2}$ as a phantom), and then divided by the administered radioactivity to obtain an image ROI-derived percentage injected dose per gram of tissue (\%ID/g). Three U87MG tumor-bearing mice were co-injected with $10 \mathrm{mg} / \mathrm{kg}$ dose of cRGD and ${ }^{64} \mathrm{Cu}$-labeled cRGD-conjugated SPIO nanocarriers to evaluate the receptor specificity of the cRGD-conjugated SPIO nanocarriers in vivo (i.e., the blocking experiment).

To anatomically localize the tracer signal obtained using PET, a few animals were also subjected to microCT scans. Right before the PET scans, animals were transported to the microCT scanning gantry, positioned, and scanned at a voxel resolution of $97 \mu \mathrm{m}$ (scanning time:7 min). Fiducial markers were used for co-registration and images were reconstructed using the built-in software (Inveon Acquisition Workshop; Siemens). The microCT and microPET datasets were loaded into IRW and fiducial markers were co-registered for alignment of datasets.

\subsection{Biodistribution studies}

Biodistribution studies were carried out on a subset of animals to confirm that the quantitative tracer uptake values based on micro PET imaging were truly representative of the actual tracer distribution in tumor-bearing mice. After the last PET scans at $48 \mathrm{~h}$ p.i., mice were euthanized. Blood, U87MG tumor, and major organs/tissues were collected and wet weighted. The radioactivity in the tissue was measured using a gamma-counter (Perkin Elmer) and presented as \% ID/g (mean $\pm \mathrm{SD})$.

\section{Results and Discussion}

\subsection{Synthesis and characterization of the SPIO nanocarriers}

The SPIO nanocarriers were prepared according to the Scheme 1. First, monodisperse SPIO NPs (10 $\mathrm{nm}$ in diameter) were prepared by one-pot high temperature reductive decomposition of $\mathrm{Fe}(\mathrm{acac})_{3}$ in oleyamine and benzyl ether using a previously published procedure [25]. The monodisperse SPIO NPs were coated with a layer of oleate and oleyamine and were only soluble in hexane and other nonpolar or weakly polar organic solvents. As shown in Figure 1a, the hydrophobic SPIO NPs were spherical and had a uniform particle size with an average diameter of $10 \mathrm{~nm}$, which was in accordance with the particle size distribution measured by dynamic light scattering (DLS, Figure 1b).

For biomedical applications, it is necessary to make the SPIO NPs hydrophilic and welldispersed in aqueous solutions. The most commonly used high affinity binding agent to stabilize SPIO NPs in water and physiologic buffers is dopamine [30,31]. However, the stability of dopamine as a high affinity anchor for SPIO NPs has been questioned recently because (1) dopamine degradation upon adsorption on SPIO NPs was reported [32] and (2) aliquots of dopamine were also shown to be prone to oxidation [33]. Therefore, we used nitrodopamine (NDA) as an anchor to immobilize the hydrophobic SPIO NPs since NDA was reported to have irreversible high affinity binding under physiologic conditions [34]. 
The NDA-capped SPIO NPs were prepared in a solvent mixture of DMF and $\mathrm{CHCl}_{3}$ under sonication, and were subsequently purified by centrifugation and dialysis against DI water. The numbers of NDA anchored onto the SPIO NPs was estimated to be 1820 based on weight analysis.

To functionalize the SPIO NPs, NHS (N-hydroxysuccinimide) ester of mono-ethyl malonate (MEM-NHS) was used as a linker for DOX conjugation; the heterobifunctional NHS$\mathrm{PEG}_{114}$-MAL (MAL denotes maleimide) was used for cRGD and NOTA conjugation; and methoxy-PEG 114 -NHS was used to adjust the resulting SPIO nanocarriers' solubility/ stability in physiological conditions and to help control the molar ratio of cRGD and NOTA that were conjugated onto the SPIO NPs via an amidation reaction (Scheme 2). The molar ratio of MEM and PEG was set at 1:1 in order to maximize the drug loading level while ensuring good solubility and stability of the resulting SPIO nanocarriers in physiological conditions. Since the number of NDA anchored onto each SPIO NP was estimated to be 1820, the total number of $\mathrm{PEG}_{114}$ conjugations per SPIO NP was estimated to be 910 . Furthermore, the molar ratio of cRGD for active tumor-targeting and that of the NOTA for isotope chelation was set at $40 \%$ and $20 \%$ respectively based on the total number of PEG arms. The reaction was carried out in DI water under a $\mathrm{N}_{2}$ atmosphere. After purification, the reaction solution was freeze dried.

DOX molecules were conjugated onto the SPIO NPs via a two-step reaction procedure [35]. Briefly, in the first step, the methoxy group of MEM was substituted with hydrazide by an ester-amide exchange aminolysis reaction. The resulting hydrazine modified SPIO NPs were purified by extensive dialysis against $0.25 \%$ ammonia solution for $48 \mathrm{~h}$ followed by freezedrying. In the second step, DOX was conjugated to the hydrazide group of the SPIO NP through an acid-sensitive hydrazone linkage. The unbound DOX was removed first by dialysis against DI water and then purified by a Sephadex LH-20 gel column.

Lastly, cRGD and NOTA were conjugated onto the distal ends of the MAL-PEG arms through a reaction between the MAL groups of PEG and the thiol groups of cRGD and thiol-functionalized NOTA (NOTA-SH). NOTA-SH was prepared by a reaction between the isothiocyanate (NCS) group of p-SCN-Bn-NOTA and the amino group of 2-

aminoethanethiol hydrocholoride in the presence of triethanolamine. Figure 1c shows the TEM image of the resulting SPIO nanocarriers with an average diameter of approximately $10 \mathrm{~nm}$. However, the hydrodynamic diameter of the SPIO nanocarriers measured by DLS was $68 \pm 2 \mathrm{~nm}$ (Figure 1d). This can be attributed to the fact that TEM only measures the diameter of the dry nanocarriers while DLS measures the hydrodynamic diameter of the SPIO nanocarriers that were functionalized with water soluble PEG (Mw: $5000 \mathrm{Da}$ ) molecules. SPIO nanocarriers without cRGD conjugation were also prepared in a similar fashion as a control. In the remaining text, they will be referred to as "cRGD-conjugated SPIO nanocarriers" and "cRGD-free SPIO nanocarriers", respectively.

The DOX and Fe loading content of the cRGD-conjugated SPIO nanocarriers as determined by UV-Vis spectroscopy (for DOX) and atomic absorption spectroscopy (AAS; for Fe) was 5.8 and $11.2 \mathrm{wt} \%$, respectively. Figure 2 shows the DOX release profiles of the cRGDconjugated SPIO nanocarriers at $\mathrm{pH} 5.3$ and 7.4. At $\mathrm{pH}$ 7.4, a negligible amount of DOX release was observed after three days. This is a desirable characteristic because during circulation in the bloodstream (where the $\mathrm{pH}$ value is $\sim 7.4$ ), DOX conjugated onto the drug nanocarriers will not be released prematurely before the nanocarriers reach the targeted tumor sites. However, at pH 5.3, a two-stage release profile was observed. Specifically, an initial rapid release stage occurred during the first $12 \mathrm{~h}$ followed by a more sustained release stage. This pH-dependent DOX release behavior is highly desirable for targeted cancer therapy because it can significantly minimize the amount of premature drug release during 
circulation in the bloodstream ( $\mathrm{pH}$ 7.4), yet provide a sufficient amount of drug to effectively kill the cancer cells once the nanocarriers are internalized and enter the endocytic pathway ( $\mathrm{pH} \sim 4.5$ to 6.5 ).

\subsection{Cellular uptake and distribution of the SPIO nanocarriers}

To investigate the cellular uptake and intracellular distribution of the SPIO nanocarriers, flow cytometry and confocal laser scanning microscopy (CLSM) were performed using the human U87MG glioblastoma cell line, which take advantage of the intrinsic fluorescence of DOX. Figure 3 shows the quantitative flow cytometry results of the cellular uptake of the cRGD-conjugated and cRGD-free SPIO nanocarriers compared to free DOX at an early (15 $\mathrm{min})$ and late (120 $\mathrm{min})$ time points. Cells without any DOX treatment were used as a negative control and showed only low-level autofluorescence at both time points. After 15 min, U87MG cells treated with cRGD-conjugated SPIO nanocarriers showed the same level of uptake as free DOX, which was 50\% higher than that of cRGD-free SPIO nanocarriers. After $120 \mathrm{~min}$, all treatment groups showed a marked increase in the level of uptake with cells treated with free DOX exhibiting the highest level of fluorescence. Quantitatively, the DOX fluorescence intensity of cells treated with free DOX, cRGD-conjugated and cRGDfree SPIO nanocarriers for 120 min were 3.6, 2.1, and 1.6-fold higher than those treated for 15 min, respectively.

Importantly, even after 120 min of treatment, cells treated with cRGD-conjugated SPIO nanocarriers still showed a $\sim 90 \%$ higher level of cellular uptake than cells treated with the cRGD-free SPIO nanocarriers. Thus, cRGD conjugation clearly increased the uptake of the SPIO nanocarriers relative to the cRGD-free nanocarriers by the $\alpha_{V} \beta_{3}$ integrin-expressing U87MG cells. The finding that cells treated with free DOX exhibited the highest level of cellular uptake is not unexpected since the free DOX can readily diffuse across the cell membrane while the cRGD-conjugated and cRGD-free SPIO nanocarriers are taken up by receptor-mediated and non-specific endocytosis, respectively [36].

To further examine the effects of cRGD conjugation on the cellular uptake of the SPIO nanocarriers, CLSM analysis of U87MG cells was performed which allows qualitative determination of the intracellular localization of the DOX fluorescence. As expected, treatment of U87MG cells with free DOX for 120 min resulted in strong DOX fluorescence both in the cytoplasm and nucleus of the cell (Figure 4). In contrast, cells treated with the cRGD-conjugated SPIO nanocarriers showed strong DOX fluorescence in the cytoplasm but lower fluorescence in the nucleus. Cells treated with the cRGD-free SPIO nanocarriers showed only low-level cytoplasmic and nuclear DOX fluorescence. Therefore, the CLSM results confirmed the findings from flow cytometry analysis, namely that cRGD-conjugation markedly increases the cellular uptake of the SPIO nanocarriers. The CLSM results further demonstrated a difference in the intracellular localization of the DOX fluorescence, i.e. free DOX can rapidly diffuse across both the cell and nuclear membranes while the SPIO nanocarriers are taken up via endocytosis and are still mostly located within the cytoplasmic endocytic compartment at the 120 min time point. Since most of the cytotoxic effects of DOX are due to its ability to bind to nuclear DNA and interrupt its function, the DOX contained within the nanocarriers must be released and exit the endocytic compartment before it can diffuse into the nucleus.

\subsection{Cytotoxicity of the SPIO nanocarriers}

To determine the effectiveness of the SPIO nanocarriers in mediating cytotoxicity against U87MG tumor cells, cells were treated with nanocarriers containing increasing concentrations of DOX, or free DOX, for 48 hours and analyzed using the MTT assay. Figure 5 shows that treatment of U87MG cells with 40 or $20 \mu \mathrm{g} / \mathrm{mL}$ free DOX had 
significantly higher cytotoxicity than treatment with either of the nanocarriers containing equivalent DOX concentrations ( $\mathrm{p}<0.05$ ). By contrast, at $10 \mu \mathrm{g} / \mathrm{mL}$ DOX, both free DOX and the cRGD-conjugated SPIO nanocarriers showed the same level of cytotoxicity, which was $\sim 20 \%$ greater than that of the cRGD-free SPIO nanocarriers ( $p<0.05$ ). Similarly, at both the $20 \mu \mathrm{g} / \mathrm{mL}$ and $40 \mu \mathrm{g} / \mathrm{mL}$ DOX concentrations, the cRGD-conjugated nanocarriers showed significantly increased levels of cytotoxicity (by $~ 20 \%$ ) than the cRGD-free SPIO nanocarriers $(\mathrm{p}<0.05)$. Together, these results demonstrate that the cRGD conjugation increases the cytotoxicity of the SPIO nanocarriers and are consistent with the increases in cellular uptake as observed with both flow cytometry and CLSM analyses. In addition, the finding that the cRGD-conjugated SPIO nanocarriers induced a significant amount of cytotoxicity in the U87MG tumor cells strongly suggests that DOX was released from the SPIO nanocarrier and entered the cell nucleus prior to this time point.

\subsection{Relaxivity measurements of the SPIO nanocarriers}

In general, MRI contrast agents are assessed via their longitudinal $\left(r_{1}\right)$ and transverse $\left(r_{2}\right)$ relaxivities, which reflect the ability of the contrast agent to alter $\mathrm{T}_{1}$ (spin-lattice relaxation time) and $\mathrm{T}_{2}$ (spin-spin relaxation time), respectively. SPIOs are generally used as $\mathrm{T}_{2}$ contrast agents. Figure 6 shows the $\mathrm{r}_{2}$ relaxivity plot of the cRGD-conjugated SPIO nanocarriers using Feridex I.V. ${ }^{\circledR}$ (an FDA approved SPIO contrast agent) as a control. Relaxivities were calculated through the least-squares curve fitting of 1/relaxation time $\left(\mathrm{s}^{-1}\right)$ versus the iron concentration $(\mathrm{mM} \mathrm{Fe})$. The $\mathrm{r}_{2}$ relaxivity $\left(18^{\circ} \mathrm{C}, 4.7 \mathrm{~T}\right)$ of the SPIO nanocarriers was $101.9 \mathrm{mM}^{-1} \mathrm{~s}^{-1}$ that was slightly lower than that of Feridex (111.5 $\mathrm{mM}^{-1} \mathrm{~s}^{-1}$ ) indicating these SPIO nanocarriers can effectively serve as $\mathrm{T}_{2}$ contrast agents.

\subsection{In Vivo investigation of SPIO nanocarriers}

Based on our previous studies with ${ }^{64} \mathrm{Cu}$-labeled nanoparticles and other recent publications on ${ }^{64} \mathrm{Cu}$-labeled SPIO NPs [37-40], the time points of $0.5,3,6,24$, and $48 \mathrm{~h}$ post-injection (p.i.) were chosen for PET scans after intravenous injection of ${ }^{64} \mathrm{Cu}$-labeled SPIO nanocarriers. The coronal slices that contained the tumors are shown in Fig. 7a and representative microPET/microCT fused images of a mouse at $6 \mathrm{~h}$ p.i. of ${ }^{64} \mathrm{Cu}$-labeled cRGD-conjugated SPIO nanocarriers are shown in Fig. 7b. The uptake and accumulation of the ${ }^{64} \mathrm{Cu}$-labeled cRGD-conjugated SPIO nanocarriers occurred primarily in the tumor and liver but not in most normal tissues, thereby demonstrating good tumor targeting capability along with good tumor contrast. Quantitative data obtained from region-of-interest (ROI) analysis are shown in Fig. 8a-c. The uptake of ${ }^{64} \mathrm{Cu}$-labeled cRGD-conjugated SPIO nanocarriers in the liver (due to the uptake of nanoparticles in the reticuloendothelial system [RES]) was prominent at early time points and gradually declined over time. The liver uptake of ${ }^{64} \mathrm{Cu}$-labeled cRGD-conjugated SPIO nanocarriers was $14.9 \pm 5.8,11.6 \pm 3.2,9.7$ $\pm 2.7,7.1 \pm 1.4$, and $6.1 \pm 1.6$ percentage injected dose per gram of tissue $(\% \mathrm{ID} / \mathrm{g})$ at $0.5,3$, 6,24 , and 48 h p.i., respectively; while the radioactivity in the blood was $3.1 \pm 1.1,2.0 \pm$ $0.2,1.8 \pm 0.3,1.5 \pm 0.3$, and $1.6 \pm 0.1 \% \mathrm{ID} / \mathrm{g}$ at $0.5,3,6,24$, and $48 \mathrm{~h}$ p.i., respectively $(\mathrm{n}=$ 3 , Figure 8a). The tumor uptake of ${ }^{64} \mathrm{Cu}$-labeled cRGD-conjugated SPIO nanocarriers were clearly visible at $0.5 \mathrm{~h}$ p.i. and plateaued after $6 \mathrm{~h}$ p.i. $(4.3 \pm 0.6,5.5 \pm 1.9,5.6 \pm 1.7,5.4 \pm$ 2.0 , and $5.4 \pm 2.1 \% \mathrm{ID} / \mathrm{g}$ at $0.5,3,6,24$, and $48 \mathrm{~h}$ p.i. respectively; $\mathrm{n}=3$; Figure $8 \mathrm{a} \& \mathrm{c})$. Administrating a blocking dose of c(RGD) $\left(10 \mathrm{mg} / \mathrm{kg}\right.$ of mouse body weight) with ${ }^{64} \mathrm{Cu}$ labeled cRGD-conjugated SPIO nanocarriers injection reduced the tumor uptake significantly $(<2 \% \mathrm{ID} / \mathrm{g}$ at all time points examined, Figure $8 \mathrm{c})$, which confirmed the integrin $\alpha_{v} \beta_{3}$ specificity of the cRGD-conjugated SPIO nanocarriers in vivo.

${ }^{64} \mathrm{Cu}$-labeled cRGD-free SPIO nanocarriers were used as a control to investigate the uptake of the SPIO nanocarriers in U87MG tumors due to passive targeting only (i.e., via the enhanced permeability and retention effect). As can be seen in Figure 8b-d, the uptake 
of ${ }^{64} \mathrm{Cu}$-labeled cRGD-free SPIO nanocarriers $(\sim 2.5 \% \mathrm{ID} / \mathrm{g})$ was significantly lower than those of ${ }^{64} \mathrm{Cu}$-labeled cRGD-conjugated SPIO nanocarriers at all time points examined ( $\mathrm{p}$ $0.05 ; n=3$ ), which further confirmed the integrin $\alpha_{\mathrm{v}} \beta_{3}$ specificity of the cRGD-conjugated SPIO nanocarriersin vivo.

After the last PET scans at $48 \mathrm{~h}$ p.i., the mice were euthanized. The tissues were collected for biodistribution studies to further validate the in vivo PET data (Figure 8d). The uptake of the cRGD-conjugated SPIO nanocarriers in the tumor was lower than that in the liver but higher than all other organs examined, indicating good tumor-targeting capability. The tumor/muscle ratio was $11.3 \pm 2.5$ at $48 \mathrm{~h}$ p.i. $(\mathrm{n}=3)$. A comparison of the biodistribution data of ${ }^{64} \mathrm{Cu}$-labeled cRGD-conjugated SPIO nanocarriers and cRGD-free SPIO nanocarriers at $48 \mathrm{~h}$ p.i. revealed that the uptake of ${ }^{64} \mathrm{Cu}$-labeled cRGD-free SPIO nanocarriers was higher in most organs except the U87MG tumor (Figure 8d), which again indicated the tumor specificity of ${ }^{64} \mathrm{Cu}$-labeled cRGD-conjugated SPIO nanocarriers. Overall, the quantification results obtained from biodistribution studies and PET scans matched well, confirming that quantitative ROI analysis of non-invasive microPET scans truly reflected the distribution of ${ }^{64} \mathrm{Cu}$-labeled SPIO nanocarriers in vivo.

\section{Conclusions}

Multifunctional and water-soluble SPIO nanocarriers were designed, synthesized and characterized for both tumor-targeted drug delivery and PET/MR imaging. These SPIO nanocarriers had a hydrodynamic diameter of $68 \pm 2 \mathrm{~nm}$ and exhibited $\mathrm{pH}$-sensitive drug release behavior. Flow cytometry and CLSM analysis demonstrated that cRGD-conjugated SPIO nanocarriers exhibited higher cellular uptake leading to higher cytotoxicity. cRGDconjugated SPIO nanocarriers also had a much higher level of tumor accumulation compared to cRGD-free SPIO nanocarriers according to in vivo PET imaging and biodistribution analyses. The MRI $r_{2}$ relaxivity of the SPIO nanocarriers were also measured which was similar to that of the Feridex, an FDA approved SPIO-based MRI contrast agent. These multifunctional SPIO nanocarriers have the potential for combined tumor-targeting drug delivery and PET/MR imaging thereby making cancer theranostics possible.

\section{Acknowledgments}

This work is supported, in part, by the National Science Foundation (DMR 1032187), Wisconsin Partnership Program, the University of Wisconsin Carbone Cancer Center, University of Wisconsin ICTR Program (NCRR 1UL1RR025011), a Susan G. Komen Postdoctoral Fellowship (to H. Hong), and a DOD PCRP IDEA Award (to W. Cai).

\section{References}

1. Allen TM, Cullis PR. Drug delivery systems: Entering the mainstream. Science. 2004; 303:18181822. [PubMed: 15031496]

2. Bawa R. NanoBiotech 2008: Exploring global advances in nanomedicine. Nanomedicine. 2009; 5:5-7. [PubMed: 19230083]

3. Tsukamoto E, Ochi S. PET/CT today: system and its impact on cancer diagnosis. Ann Nucl Med. 2006; 20:255-267. [PubMed: 16856569]

4. Peer D, Karp JM, Hong S, Farokhzad OC, Margalit R, Langer R. Nanocarriers as an emerging platform for cancer therapy. Nature Nanotech. 2007; 387:751-760.

5. Davis ME, Chen Z, Shin DM. Nanoparticle therapeutics: an emerging treatment modality for cancer. Nature Reviews. 2008; 7:771-782.

6. Petros RA, DeSimone JM. Strategies in the design of nanoparticles for therapeutic applications. Nature Reviews. 2010; 9:615-627. 
7. Louie A. Multimodality imaging probes: design and challenges. Chem Rev. 2010; 110:3146-3195. [PubMed: 20225900]

8. Liong M, Lu J, Kovochich M, Xia T, Ruehm SG, Nel AE, et al. Multifunctional inorganic nanoparticles for imaging, targeting, and drug delivery. ACS Nano. 2008; 2:889-896. [PubMed: 19206485]

9. Kim J, Lee JE, Lee SH, Yu JH, Lee JH, Park TG, et al. Designed fabrication of a multifunctional polymer nanomedical platform for simultaneous cancer-targeted imaging and magnetically guided drug delivery. Adv Mater. 2008; 20:478-483.

10. Peer D, Kar JM, Hong S, Farokhzad OC, Margalit R, Langer R. Nanocarriers as an emerging platform for cancer therapy. Nat Nanotechnol. 2007; 2:751-760. [PubMed: 18654426]

11. Zhang L, Chan JM, Gu FX, Rhee JW, Wang AZ, Radovic-Moreno AF, et al. Self-assembled lipidPpolymer hybrid nanoparticles: a robust drug delivery platform. ACS Nano. 2008; 2:1696-1702. [PubMed: 19206374]

12. Beyer T, Townsend DW, Blodgett TM. Dual-modality PET/CT tomography for clinical oncology. Q J Nucl Med. 2002; 46:24-34. [PubMed: 12072843]

13. Blodgett TM, Meltzer CC, Townsend DW. PET/CT: form and function. Radiology. 2007; 242:360-365. [PubMed: 17255408]

14. Townsend DW. Dual-modality imaging combining anatomy and function. J Nucl Med. 2008; 49:938-955. [PubMed: 18483101]

15. Judenhofer MS, Wehrl HF, Newport DF, Catana C, Siegel SB, Becker M, et al. Simultaneous PETMRI: a new approach for functional and morphological imaging. Nat Med. 2008; 14:459-465. [PubMed: 18376410]

16. Glaus C, Rossin R, Welch MJ, Bao G. In vivo evaluation of ${ }^{64} \mathrm{Cu}$-labeled magnetic nanoparticles as a dual-modality PET/MR imaging agent. Bioconjugate Chem. 2010; 21:715-722.

17. Pichler BJ, Wehrl HF, Kolb A, Judenhofer MS. Positron emission tomography/magnetic resonance imaging: the next generation of multimodality imaging? Semin Nucl Med. 2008; 38:199-208. [PubMed: 18396179]

18. Wehrl HF, Judenhofer MS, Wiehr S, Pichler BJ. Pre-clinical PET/MR: technological advances and new perspectives in biomedical research. Eur J Nucl Med Mol Imaging. 2009; 36:S56-68. [PubMed: 19194703]

19. Glaus C, Rossin R, Welch MJ, Bao G. In vivo evaluation of ${ }^{64} \mathrm{Cu}$-labeled magnetic nanoparticles as a dual-modality PET/MR imaging agent. Bioconjugate Chem. 2010; 21:715-722.

20. Cai W, Chen X. Nanoplatforms for targeted molecular imaging in living subjects. Small. 2007; 3:1840-1854. [PubMed: 17943716]

21. Hong H, Zhang Y, Sun J, Cai W. Molecular imaging and therapy of cancer with radiolabeled nanoparticles. Nano Today. 2009; 4:399-413. [PubMed: 20161038]

22. Sosnovik DE, Weissleder R. Emerging concepts in molecular MRI. Curr Opin Biotechnol. 2007; 18:4-10. [PubMed: 17126545]

23. Cai W, Chen X. Multimodality molecular imaging of tumor angiogenesis. J Nucl Med. 2008; 49:113S-28S. [PubMed: 18523069]

24. Cai W, Niu G, Chen X. Imaging of integrins as biomarkers for tumor angiogenesis. Curr Pharm Des. 2008; 14:2943-2973. [PubMed: 18991712]

25. Xie J, Xu C, Xu Z, Hou Y, Young KL, Wang SX, et al. Linking hydrophilic macromolecules to monodisperse magnetite $\left(\mathrm{Fe}_{3} \mathrm{O}_{4}\right)$ nanoparticles via trichloro-s-triazine. Chem. Mater. 2006; 18:5401-5403.

26. Napolitano A, Dischia M, Constantini C, Prota GA. A new oxidation pathway of the neurotoxin 6aminodopamine. Isolation and characterisation of a dimer with a tetrahydro(3,4a)iminoethanophenoxazine ring system. Tetrahedron. 1992; 48:8515-8522.

27. Ranganathan S, Tamilarasu N. Cheminform abstract: the transformation of dihydroxyphenylalanine to metal uptake systems and ionophores. J Indian Chem Soc. 1999; 76:727-731.

28. Cai W, Wu Y, Chen K, Cao Q, Tice DA, Chen X. In vitro and in vivo characterization of ${ }^{64} \mathrm{Cu}-$ labeled Abegrin ${ }^{\mathrm{TM}}$, a humanized monoclonal antibody against integrin $\alpha_{\mathrm{v}} \beta_{3}$. Cancer Res. 2006; 66:9673-9681. [PubMed: 17018625] 
29. Cai W, Chen K, Mohamedali KA, Cao Q, Gambhir SS, Rosenblum MG, et al. PET of vascular endothelial growth factor receptor expression. J Nucl Med. 2006; 47:2048-2056. [PubMed: 17138749]

30. Gu HW, Yang ZM, Gao JH, Chang CK, Xu B. Heterodimers of nanoparticles: formation at a liquid-liquid interface and particle-specific surface modification by functional molecules. J Am Chem Soc. 2005; 127:34-35. [PubMed: 15631435]

31. Xu CJ, Xu KM, Gu HW, Zheng RK, Liu H, Zhang XX, et al. Dopamine as a robust anchor to immobilize functional molecules on the iron oxide shell of magnetic nanoparticles. J Am Chem Soc. 2004; 126:9938-9939. [PubMed: 15303865]

32. Shultz MD, Reveles JU, Khanna SN, Carpenter EE. Reactive nature of dopamine as a surface functionalization agent in iron oxide nanoparticles. J Am Chem Soc. 2007; 129:2482-2487. [PubMed: 17290990]

33. Zürcher S, Wacherlin D, Bethuel Y, Malisova B, Textor M, Tosatti S, et al. Biomimetic surface modification based on the cyanobacterial iron chelator anachelin. J Am Chem Soc. 2006; 128:1064-1065. [PubMed: 16433508]

34. Amstad E, Gillich T, Bilecka I, Textor M, Reimhult E. Ultrastable iron oxide nanoparticle colloidal suspensions using dispersants with catechol-derived anchor groups. Nano Letters. 2009; 9:40424048. [PubMed: 19835370]

35. Aryal S, Grailer JJ, Pilla S, Steeber DA, Gong S. Doxorubicin conjugated gold nanoparticles as water-soluble and pH-responsive anticancer drug nanocarriers. J Mater Chem. 2009; 19:78797884.

36. Yang X, Grailer JJ, Rowland IJ, Javadi A, Hurley SA, Steeber DA, Gong S. Multifunctional SPIO/ DOX-loaded wormlike polymer vesicles for cancer therapy and MR imaging. Biomaterials. 2010; 31:9065-9073. [PubMed: 20828811]

37. Cai W, Chen K, Li ZB, Gambhir SS, Chen X. Dual-function probe for PET and near-infrared fluorescence imaging of tumor vasculature. J Nucl Med. 2007; 48:1862-1870. [PubMed: 17942800]

38. Lee HY, Li Z, Chen K, Hsu AR, Xu C, Xie J, et al. PET/MRI dual-modality tumor imaging using arginine-glycine-aspartic (RGD)-conjugated radiolabeled iron oxide nanoparticles. J Nucl Med. 2008; 49:1371-1379. [PubMed: 18632815]

39. Liu Z, Cai W, He L, Nakayama N, Chen K, Sun X, et al. In vivo biodistribution and highly efficient tumour targeting of carbon nanotubes in mice. Nat Nanotechnol. 2007; 2:47-52. [PubMed: 18654207]

40. Xie J, Chen K, Huang J, Lee S, Wang J, Gao J, et al. PET/NIRF/MRI triple functional iron oxide nanoparticles. Biomaterials. 2010; 31:3016-3022. [PubMed: 20092887] 

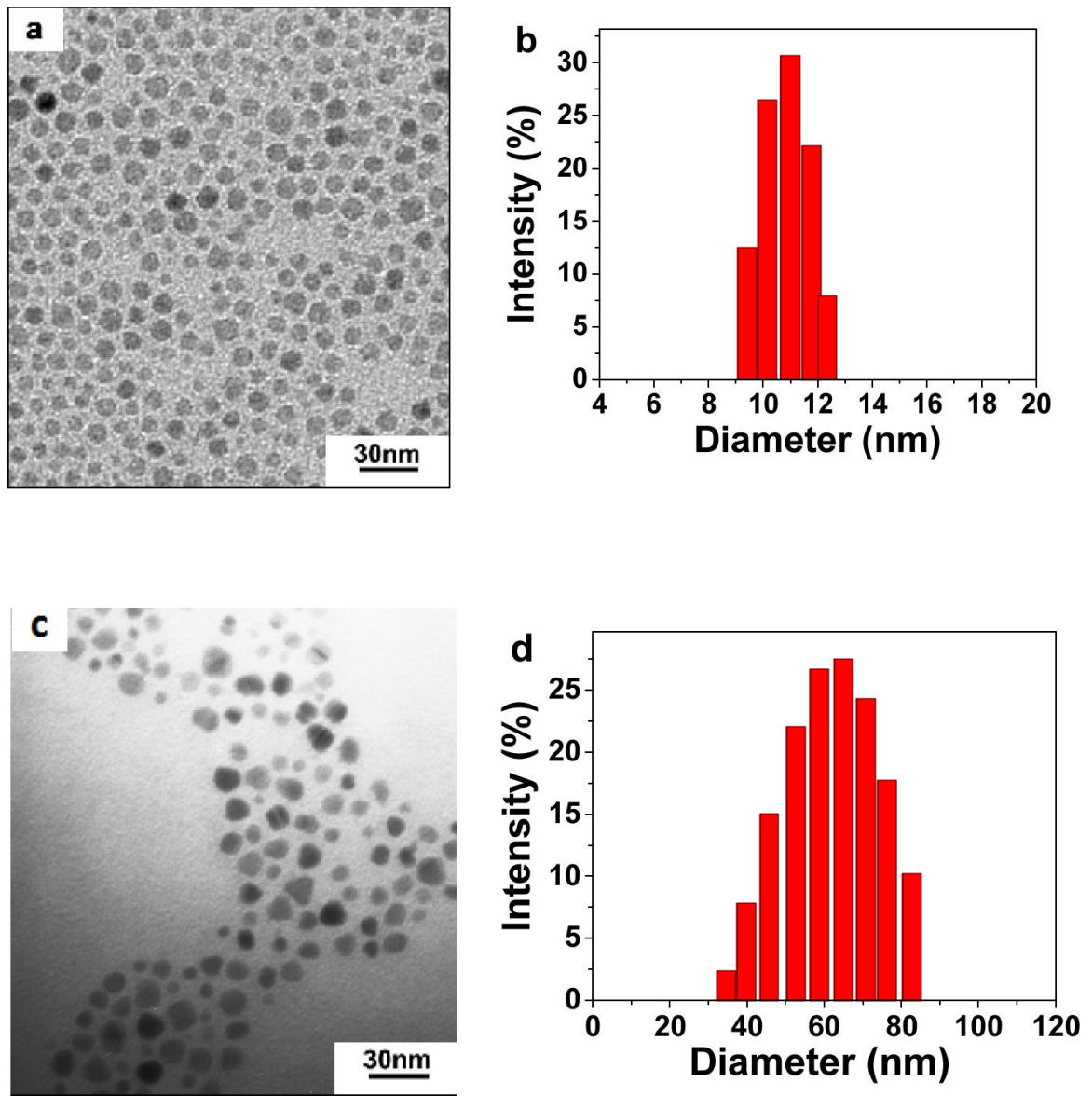

Figure 1.

TEM image (a) and DLS evaluation (b) of $10 \mathrm{~nm}$ hydrophobic SPIO NPs in hexane; TEM image (c) and DLS evaluation (d) of cRGD-conjugated SPIO nanocarriers. 


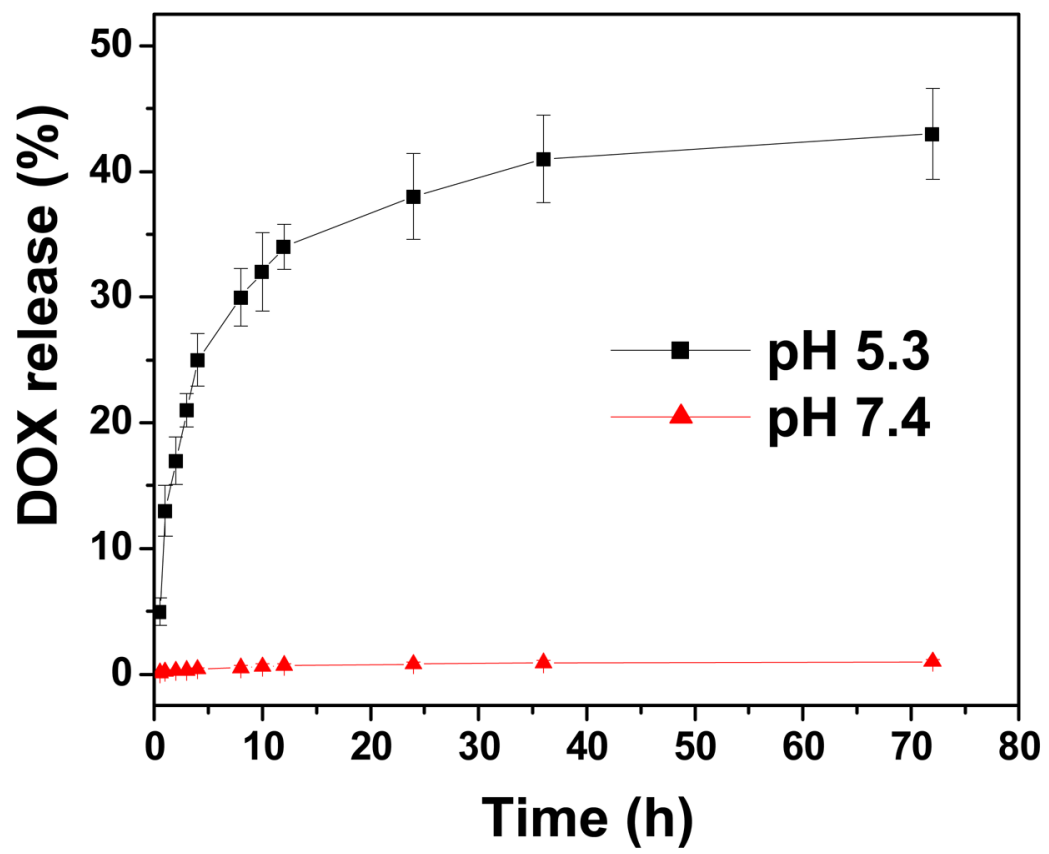

Figure 2.

DOX release profile of cRGD-conjugated nanocarriers at $\mathrm{pH} 5.3$ and 7.4. 


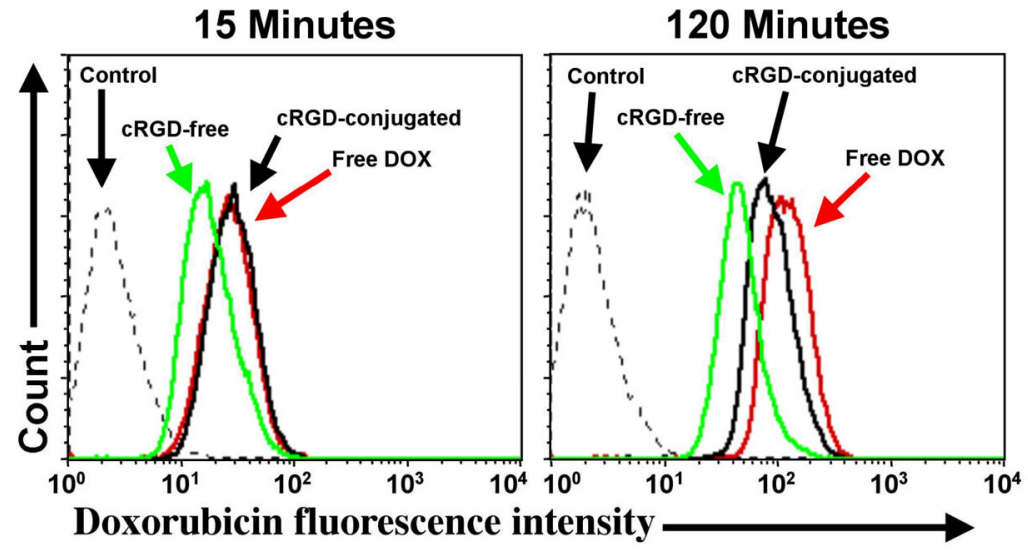

Figure 3.

Flow cytometry results of U87MG cells treated with free DOX, cRGD-conjugated or cRGDfree SPIO nanocarriers (DOX concentration, $20 \mu \mathrm{g} / \mathrm{mL}$ ) or medium alone (control) for 15 and 120 minutes at $37^{\circ} \mathrm{C}$. 

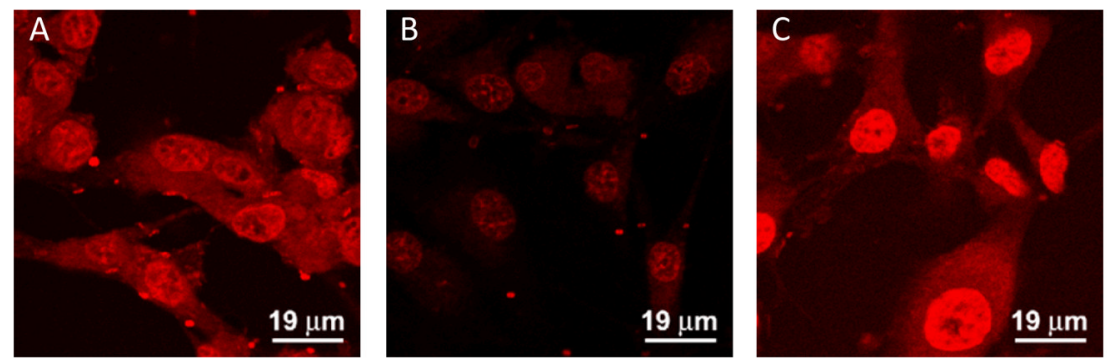

Figure 4.

CLSM images of U87MG cells treated with (A) cRGD-conjugated SPIO nanocarriers; (B) cRGD-free SPIO nanocarriers; or (C) free DOX at $37^{\circ} \mathrm{C}$ for 120 minutes (DOX concentration, $20 \mu \mathrm{g} / \mathrm{mL}$ ). 


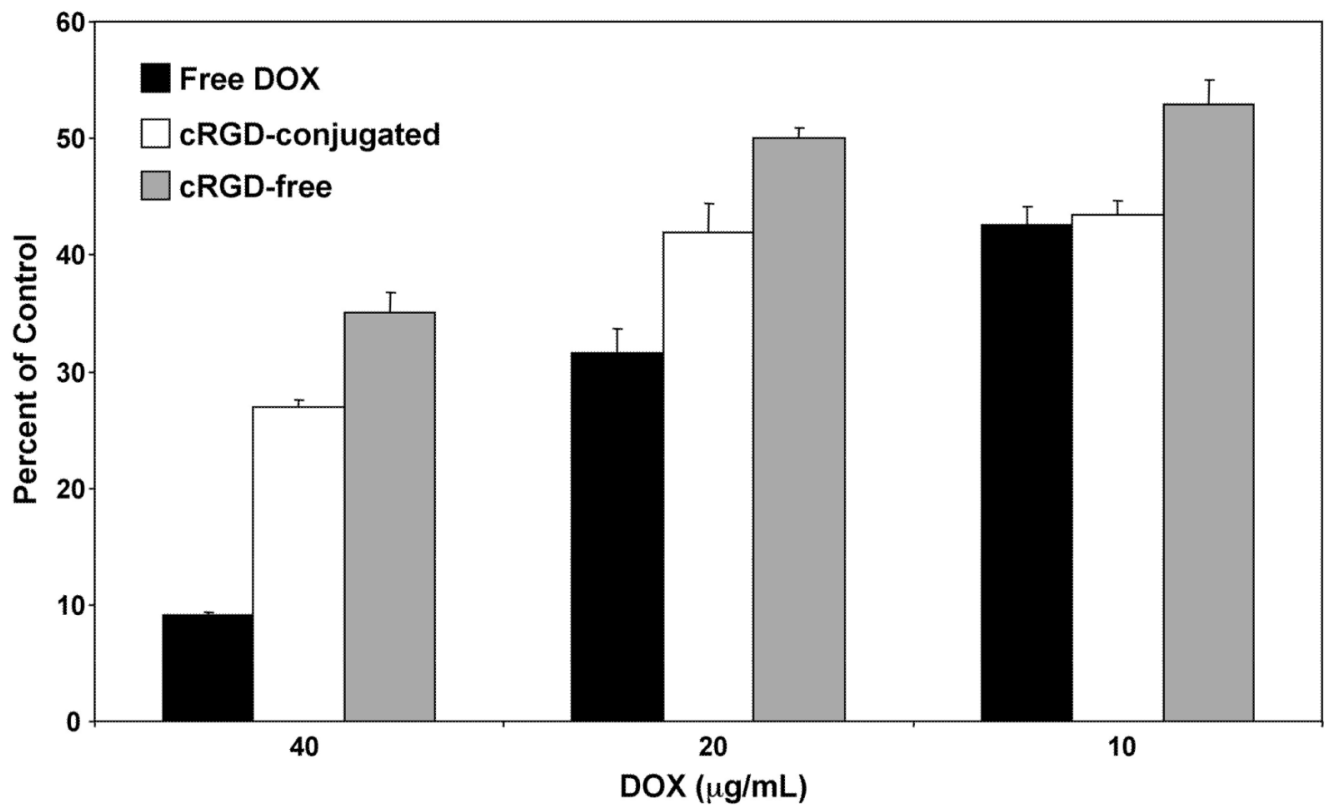

Figure 5.

Cytotoxicity of U87MG cells treated with free DOX, cRGD-conjugated, or cRGD-free SPIO nanocarriers for 48 hours at different DOX concentrations. Following the 48 hour treatment the degree of cytotoxicity was determined using the MTT assay. 


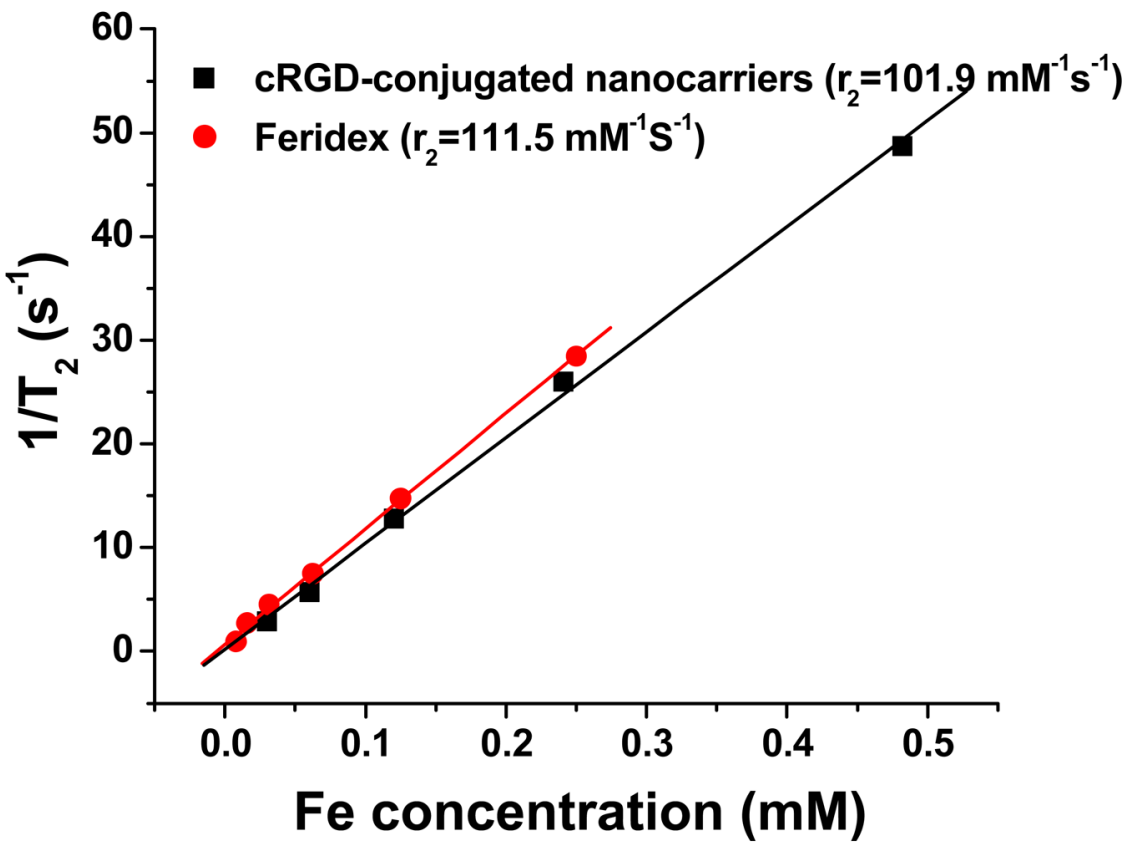

Figure 6.

$\mathrm{T}_{2}$ relaxation rates $\left(1 / \mathrm{T}_{2}, \mathrm{~s}^{-1}\right)$ as a function of iron concentration (mM) for both cRGDconjugated SPIO nanocarriers and commercial Feridex ${ }^{\circledR}$. 
a

CRGDconjugated SPIO nanocarrier
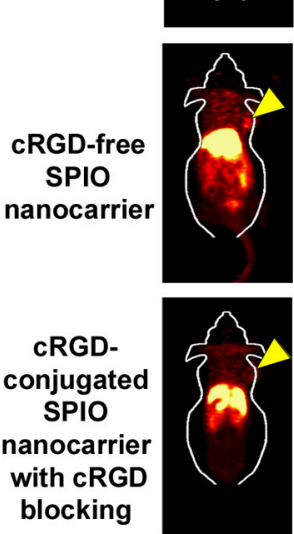

$0.5 \mathrm{~h}$
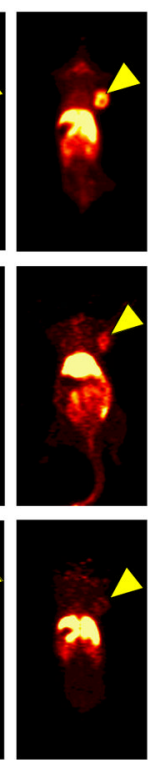

$3 \mathrm{~h}$
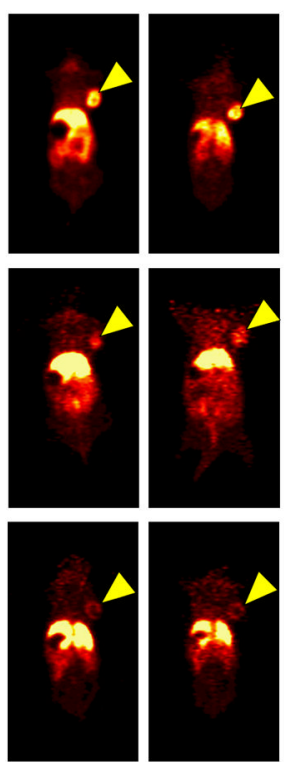

$6 \mathrm{~h}$

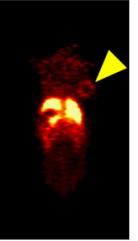

$24 \mathrm{~h}$

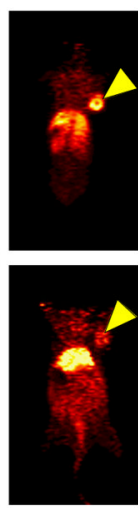

$6 \% \mathrm{ID} / \mathrm{g}$

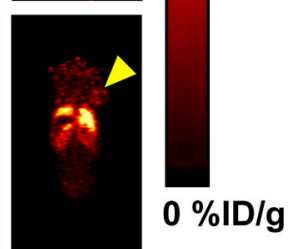

$48 \mathrm{~h}$

b

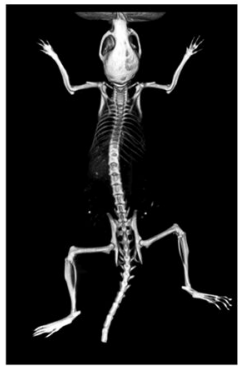

CT

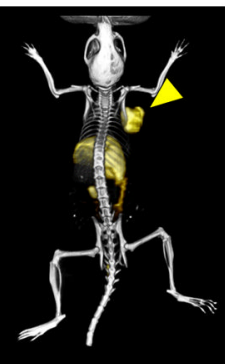

PET/CT

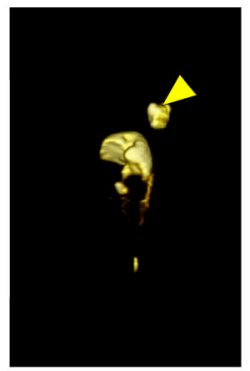

PET

Figure 7.

In vivo investigation of ${ }^{64} \mathrm{Cu}$-labeled SPIO nanocarriers. (a) Serial coronal PET images of U87MG tumor-bearing mice at various time points post-injection of ${ }^{64} \mathrm{Cu}$-labeled SPIO nanocarriers (cRGD-conjugated, cRGD-free, and cRGD-conjugated with a blocking dose of cRGD). (b) Representative PET/CT images of a U87MG tumor-bearing mouse at $6 \mathrm{~h}$ postinjection of ${ }^{64} \mathrm{Cu}$-labeled cRGD-conjugated SPIO nanocarriers. 

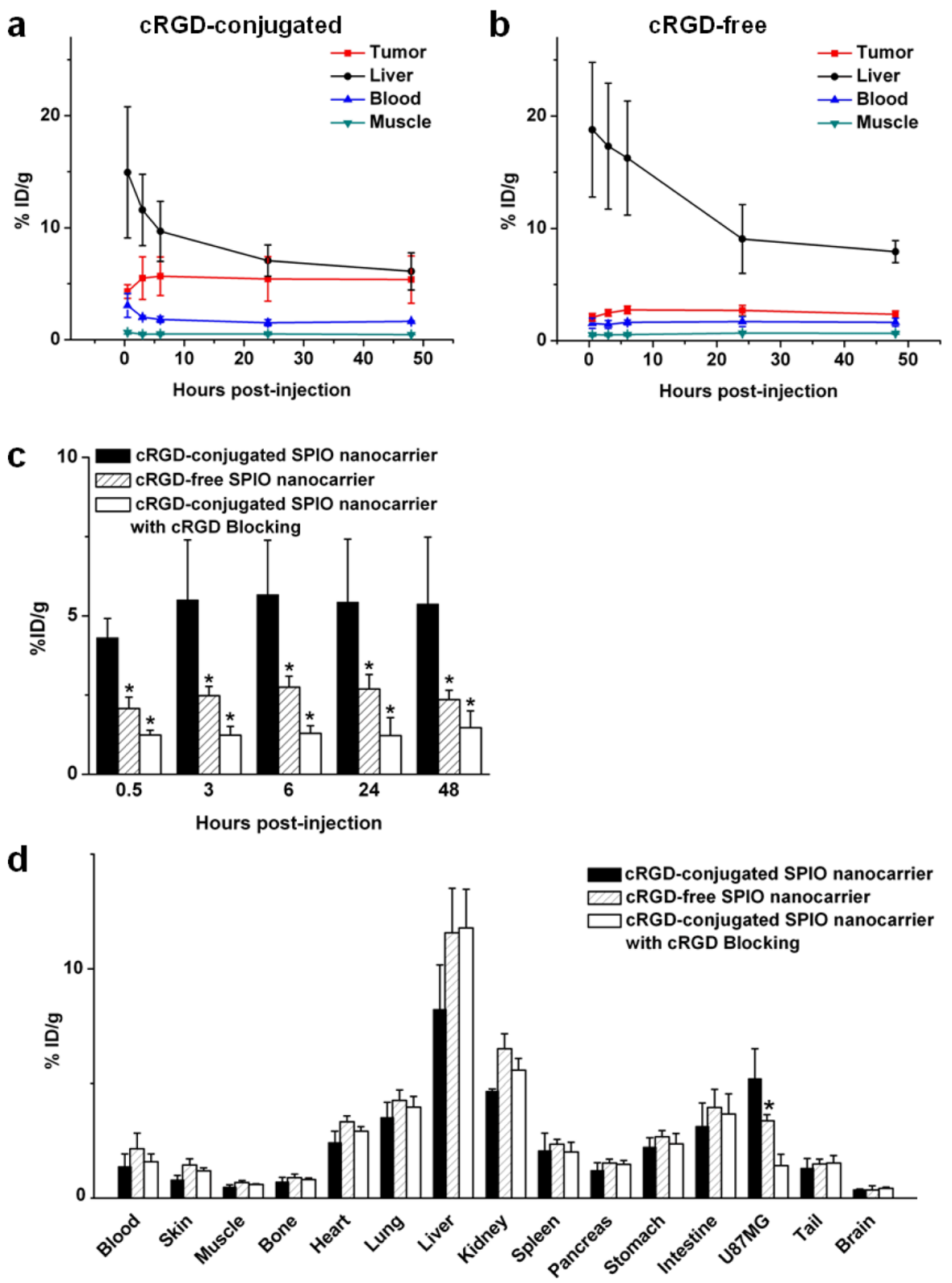

Figure 8.

ROI analysis and biodistribution studies. (a) Time-activity curves of the U87MG tumor, liver, blood, and muscle upon intravenous injection of ${ }^{64} \mathrm{Cu}$-labeled cRGD-conjugated SPIO nanocarriers $(\mathrm{n}=3)$. (b) Time-activity curves of the U87MG tumor, liver, blood, and muscle upon intravenous injection of ${ }^{64} \mathrm{Cu}$-labeled cRGD-free SPIO nanocarriers $(n=3)$. (c) Comparison of U87MG tumor uptake of ${ }^{64} \mathrm{Cu}$-labeled SPIO nanocarriers (cRGDconjugated, cRGD-free, and cRGD-conjugated with a blocking dose of cRGD, $n=3$ ). (d) Biodistribution of ${ }^{64} \mathrm{Cu}$-labeled SPIO nanocarriers (cRGD-conjugated, cRGD-free, and cRGD-conjugated with a blocking dose of $\mathrm{cRGD}, \mathrm{n}=3$ ) at $48 \mathrm{~h}$ post-injection. *: $\mathrm{p}<0.05$. 


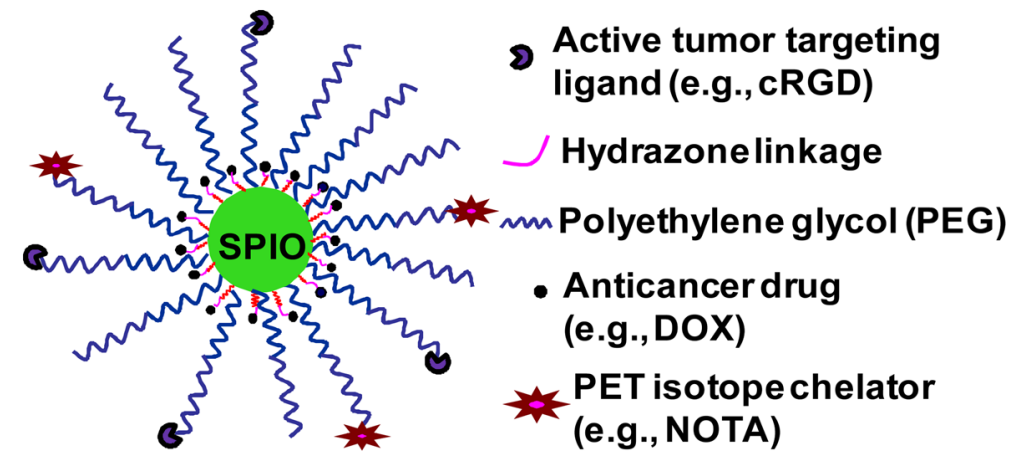

Scheme 1.

Illustration of the multifunctional cRGD-conjugated SPIO nanocarriers for combined tumortargeting drug delivery and PET/MR imaging. 

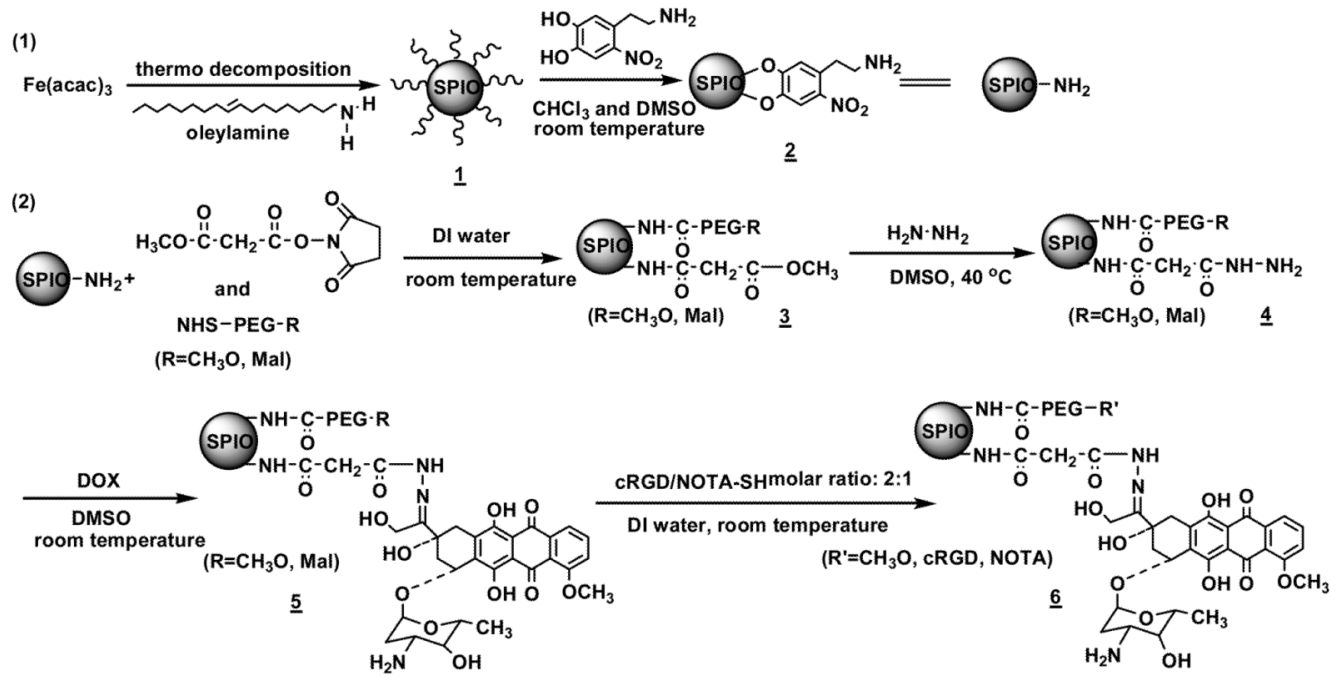

Scheme 2.

Procedures used to prepare the multifunctional SPIO nanocarriers. 